THE STEFAN PROBLEM WITH SURFACE TENSION IN THE THREE DIMENSIONAL CASE WITH SPHERICAL SYMMETRY: NON-EXISTENCE

OF THE CLASSICAL SOLUTION

By

A.M. Meirmanov

IMA Preprint Series \# 799

May 1991 


\title{
THE STEFAN PROBLEM WITH SURFACE TENSION IN THE THREE DIMENSIONAL CASE WITH SPHERICAL SYMMETRY: NON-EXISTENCE OF THE CLASSICAL SOLUTION
}

\author{
A.M. MEIRMANOV
}

\begin{abstract}
The Stefan problem wi th surface tension in three dimensional case with spherical symmetry is considered. We first establish the existence and uniqueness of the classical solution of the Stefan problem with surface tension and kinetic undercooling effects

$$
\theta=\theta_{*}-\gamma k+\beta V_{\nu}
$$

for all time and then pass to the limit as $\beta \rightarrow 0$. The limiting solution be the global-time weak solution and the local-time classical solution of the Stefan problem with surface tension. These solution cannot be the global-time classical solution. If $S(t)$ be the radius of the solid ball, then there exist time $\bar{t}_{*}>0$ such that

$$
S\left(\bar{t}_{*}+0\right)<S\left(\bar{t}_{*}-0\right) .
$$
\end{abstract}

Key words. Supercooling, Stefan problem, Gibbs-Thomson law.

AMS(MOS) subject classifications. 35R35, 35K20, 35K85.

Introduction. This work arose as an answer to a question of J.R. Ockendon and S. Howison.

The question being, how to calculate the time of disappearance of a solid ball in a supercooling liquid?

When we say "supercooling liquid", we mean that the process is modeled by the Stefan problem with surface tension.

Before giving a formulation of this problem let me recall the Stefan problem.

We have a continuous medium occupying a domain $\Omega, \theta$ is the temperature and the domains $\Omega^{+}(t), \Omega^{-}(t)$ correspond to the liquid and the solid parts of the domain $\Omega$ at the moment $t$.

In each domains $\Omega^{ \pm}(t)$ the temperature $\theta$ satisfies the heat equation

$$
C \frac{\partial \theta}{\partial t}=\kappa \Delta \theta, \vec{x} \in \Omega^{ \pm}(t), \quad t>0
$$

Let $\Gamma(t)$ be the free boundary between the liquid and the solid. We have

$$
[\theta]=0, \quad\left[\kappa \frac{\partial \theta}{\partial \nu}\right]=L \cdot V_{\nu}, \quad \vec{x} \in \Gamma(t), \quad t>0
$$


and

$$
\theta=\theta_{*}, \quad \vec{x} \in \Gamma(t), \quad t>0 .
$$

Here $c$ is a heat capacity, $\kappa$ is a heat conductivity, $[f]$ is a jump of the function $f$ on the free boundary $\Gamma(t), L$ is the latent heat (all constants are positive), $\vec{\nu}$ is a normal vector to the free boundary, $V_{\nu}$ is the normal speed of the free boundary and $\theta_{*}$ is the melting temperature.

The problem (0.1)-(0.3) is completed with the addition of initial conditions and a boundary condition on the known boundary $\partial \Omega$.

If one introduces a phase function $\varphi$, such that

$$
\varphi=1 \text { in } \Omega^{+}(t), \quad \varphi=0 \text { in } \Omega^{-}(t),
$$

then equation (0.1) together with conditions (0.2) may be rewritten in the form

$$
\frac{\partial}{\partial t}(c \theta+L \varphi)=\kappa \Delta \theta, \quad \vec{x} \in \Omega, \quad t>0
$$

Equation (0.5) together with corresponding initial and boundary conditions can be considered as a problem of finding only the temperature $\theta$. Then the condition (0.3) on the free boundary $\Gamma(t)$ is reduced to a problem of determining on the phase function $\varphi$.

If one introduces Heaviside's graph $H(\xi)$, then the condition $(0.3)$ may be rewritten in the form

$$
\varphi \in H\left(\theta-\theta_{*}\right), \quad \vec{x} \in \Omega, t>0
$$

The problem (0.5), (0.6) together with initial and boundary conditions on the boundary $\partial \Omega$ is a weak formulation of the Stefan problem.

From the physical point of view the Stefan problem is the simplest equilibrium phasetransition model, but this is not a simple problem from the mathematical point of view.

Perhaps more natural and more interesting for applications are the nonequilibrium phase-transitions models. The Stefan problem with surface tension can be the simplest model of such problems.

There are two independent thermodynamic parameters: the temperature $\theta$ and the phase function $\varphi$. Temperature $\theta$ as in the Stefan problem satisfies equation (0.5), and phase function $\varphi$, instead of equation (0.6) in the Stefan problem, satisfies the GibbsThomson law on the free boundary

$$
\theta=\theta_{*} \quad \mp \gamma k, \quad \vec{x} \in \Gamma(t), \quad t>0
$$


where positive constant $\gamma$ is the surface tension, $k$ is the mean curvature of the interface. If a line segment $x_{0} \vec{z}_{0}$ of the segment $x_{0} \vec{y}_{0}$ connecting the interface point $\vec{x}_{0}$ to the center of curvature $\vec{y}_{0}$ lies in the solid, then in (0.7) we take the negative sign.

More general form of the Gibbs-Thomson law on the free boundary is the condition

$$
\theta=\theta_{*}-k \gamma+\beta V_{\nu}, \quad \vec{x} \in \Gamma(t), \quad t>0,
$$

where a small constant $\beta$ is a positive, if a normal $\vec{\nu}$ be the outside to the solid.

A. Visintin [1] proved the existence of at least one weak solution of problem (0.5), (0.8). S. Luckhaus [2] proved the existence of a weak solution of the problem (0.5), (0.7) and showed the nonuniqueness of this solution. X. Chen and F. Reitich [3] proved the local-time existence of the classical solution of the problem (0.5), (0.8) and E. Radkevitch [4] proved the local-time existence of the classical solution of problems $(0.5),(0.7)$ and $(0.5),(0.8)$. One-phase problem with spherical symmetry is considered by J. Chadam, S. Howison and P. Ortoleva [5].

Now, let us consider a spherical solid ball in $\mathbb{R}^{3}$ surrounded by a supercooling liquid. Both the liquid and the solid are contained in the spherical domain $\Omega$ with the same center as the solid ball.

We suppose, that the initial temperature depends only on radius $x=|\vec{x}|$ and temperature on the boundary $\partial \Omega$ depends only on time $t$. Then the problem $(0.5),(0.7)$ (or (0.5), $(0.8))$ has a solution $\{\theta, \varphi\}$ depending only on the radius $x$ and the time $t$.

Firstly we make a change of variables to dimensionless form

$$
t \rightarrow t / t_{0}, \quad x \rightarrow x / x_{0}, \quad \theta \rightarrow \frac{\theta-\theta_{*}}{\theta_{0}}
$$

and choose

$$
\theta_{0}=\frac{2 \gamma}{x_{0}}, \quad t_{0}=\frac{c x_{0}^{2}}{\kappa}, \quad \lambda=\frac{L x_{0}}{2 \gamma}
$$

Then (keeping for simplicity the same notations $x, t, \theta, \varphi, \Omega, \Omega^{ \pm}(t)$ ) we obtain

$$
\begin{gathered}
\frac{\partial}{\partial t}(\theta+\lambda \varphi)=\frac{1}{x} \frac{\partial^{2}}{\partial x^{2}}(x \theta), \quad x \in \Omega, \quad t>0, \\
\theta(x, 0)=\tilde{\theta}_{0}(x), \quad \varphi(x, 0)=\varphi_{0}(x), \quad x \in \Omega, \\
\lim _{x \rightarrow 0}|\theta|<\infty, \quad \theta(\ell, t)=\theta_{1}(t), \quad t>0, \\
\theta=-\frac{1}{S(t)}, \quad x=S(t), \quad t>0,
\end{gathered}
$$

and instead of conditions $(0.5),(0.8)$ we have $(0.10),(0.11),(0.12)$ and

$$
\theta=-\frac{1}{S(t)}+\beta \frac{d S}{d t}, \quad x=S(t), t>0
$$


Here $\Omega=\{x \mid 0<x<\ell\}, \ell=$ const $>1, S(t)$ is the radius of the solid ball, $\Omega^{-}(t)=$ $\{x \mid 0<x<S(t)\}, \Omega^{+}(t)=\{x \mid S(t)<x<\ell\}$.

The main results of the present work are:

1. Existence and uniqueness of the classical solution of the problem $(0.10)-(0.12)$, $(0.14)$ for any $\beta>0$ and all time $t>0$.

2. Local-time existence and uniqueness of the classical solution of the problem (0.10)(0.13).

3. Existence of at least one weak solution of the problem (0.10) - (0.13) with monotone free boundary $x=S(t)$ for all time $t>0$.

4. Nonexistence of the classical solution of the problem (0.10)-(0.13) for all time $t>0$.

5. Nonuniqueness of the solution of the problem $(0.10)-(0.13)$ when $\varphi_{0} \equiv 0$.

Let us introduce a new unknown function

$$
u=x \cdot \theta(x, t)+1 .
$$

Then the problems $(0.10)-(0.13)$ or $(0.10)-(0.12),(0.14)$ have the form

$$
\begin{gathered}
\frac{\partial}{\partial t}(u+\lambda x \varphi)=\frac{\partial^{2} u}{\partial x^{2}}, \quad x \in \Omega, \quad t>0, \\
u(x, 0)=u_{0}(x), \quad \varphi(x, 0)=\varphi_{0}(x), \quad x \in \Omega, \\
u(0, t)=1, \quad u(\ell, t)=u^{1}(t), \quad t>0, \\
u=0, \quad x=S(t), \quad t>0,
\end{gathered}
$$

or, in the second case, we replace (0.19) with

$$
u=-\beta S \frac{d S}{d t}, \quad x=S(t), t>0
$$

The problem (0.16)-(0.19) is exactly the Stefan problem, but with a negative and nonconstant latent heat $\tilde{L}=-\lambda x$.

Actually, if the solution of the problem (0.16)-(0.19) is smooth enough, then the equation $(0.16)$ is equivalent to the heat equation in each domain $\Omega^{ \pm}(t)$

$$
\frac{\partial u}{\partial t}=\frac{\partial^{2} u}{\partial x^{2}}, \quad x \in \Omega^{ \pm}(t), \quad t>0,
$$

with the condition

$$
[u]=0, \quad x=S(t), t>0,
$$


and the Stefan condition with negative latent heat

$$
\lambda S \frac{d S}{d t}=\left[\frac{\partial u}{\partial x}\right]_{S(t)+0}^{S(t)-0}, \quad x=S(t), t>0
$$

We choose new variables such that $S(0)=1$.

If we suppose that $u^{1}(t) \equiv 0, u_{0}(x) \geq$ for $x \in(0,1), u_{0}(x) \equiv 0$ for $x \in(1, \ell), \varphi_{0}(x) \equiv 0$ for $x \in(0,1), \varphi_{0}(x) \equiv 1$ for $x \in(1, \ell)$ then we obtain a one-phase problem

$$
\begin{gathered}
\frac{\partial u}{\partial t}=\frac{\partial^{2} u}{\partial x^{2}}, \quad 0<x<S(t), \quad t>0 \\
u=0, \quad \lambda S \cdot \frac{d S}{d t}=\frac{\partial u}{\partial x}, \quad x=S(t), t>0, \\
u(0, t)=1, \quad u(x, 0)=u_{0}(x), \quad S(0)=1 .
\end{gathered}
$$

Our conclusion concerning the nonexistence of the classical solution of the Stefan problem with surface tension for all time is based upon a very simple identity; multiplying equation (0.25) by $x$ and integrating by parts, one obtains after several simple transformations

$$
\int_{0}^{S(t)} x u(x, t) d x-\frac{\lambda}{3} S^{3}(t)=\int_{0}^{1} x u_{0}(x) d x-\frac{\lambda}{3}+t .
$$

First of all note that if

$$
\lambda \leq 3 \int_{0}^{1} x u_{0}(x) d x
$$

then the radius of the solid ball $S(t)$ is strictly greater than zero as long as the classical solution exists.

Actually, the classical solution of the problem (0.24)-(0.26) satisfies the maximum principle

$$
\begin{gathered}
0<u(x, t)<\max \left\{\max u_{0}(x), 1\right\}, x \in \Omega^{-}(t), \quad t>0 \\
\frac{\partial u}{\partial x}(S(t)-0, t)<0, \quad t>0 .
\end{gathered}
$$

Applying inequality (0.29) to the identity (0.27) one has

$$
\begin{gathered}
\frac{1}{2} \max |u| \cdot S^{2}(t)=\max |u| \cdot \int_{0}^{S(t)} x d x \geq \int_{0}^{S(t)} x u(x, t) d x \geq \\
\int_{0}^{S(t)} x u(x, t) d x-\frac{\lambda}{3} S^{3}(t)=\int_{0}^{1} x u_{0}(x) d x-\lambda 3+t \geq t .
\end{gathered}
$$


So, the radius of the solid ball is strictly greater than zero for all time.

On the other hand, this radius is bounded for all time:

$$
S(t) \leq 1, \quad t>0
$$

This follows from (0.30) and Stefan condition (0.25). If one supposes now that the classical solution of the problem (0.24)-(0.26) exist for all time, then one obtains a contradiction to $(0.27)$ : the left hand side of the identity $(0.27)$ is bounded, while the right hand side increases with time $t$.

Hence, there exists a time $t_{*}$ such that

$$
\lim _{t \rightarrow t_{*}-0}\left|\frac{d S}{d t}(t)\right|=\infty
$$

It is difficult to see what happens with the solution when $t>t_{*}$. After several discussions with J. Ockendon he prompted me use an approximating law $(0.20)$ and then pass to the limit as $\beta \rightarrow 0$.

Let us follow this way. (All notations of functional spaces and norms in these spaces from $[6])$.

$\S 1$. Approximating problem (0.16)-(0.18), (0.20) $(\beta>0)$. So, one has to determine functions $u(x, t)$ and $S(t)$ such that

$$
\begin{gathered}
\frac{\partial u}{\partial t}=\frac{\partial^{2} u}{\partial x^{2}}, \quad x \in \Omega^{ \pm}(t), \quad t>0 \\
{[u]=0, \beta\left[\frac{\partial u}{\partial x}\right]_{S(t)+0}^{S(t)-0}+\lambda u=0, \quad x=S(t), t>0} \\
u(0, t)=1, \quad u(\ell, t)=u^{1}(t), \quad t>0, \\
u(x, 0)=u_{0}(x), x \in \Omega, \quad S(0)=1, \\
u=-\beta S \cdot \frac{d S}{d t}, \quad x=S(t), t>0 .
\end{gathered}
$$

We used condition (1.5) for changing the Stefan condition to the form (1.2).

First of all let us consider the linear problem (1.1)-(1.4) with given boundary $x=\bar{S}(t)$ such that

$$
\delta \leq \bar{S}(t) \leq \ell-\delta
$$

with sufficiently small positive constant $\delta$.

Denote as usual

$$
\Omega^{-}(t)=\{x \mid 0<x<\bar{S}(t)\}, \Omega^{+}(t)=\{x \mid \bar{S}<x<\ell\}, \Omega_{T}^{ \pm}=\underset{0<t<T}{\mathrm{U}} \Omega^{ \pm}(t) .
$$


Assume, that the functions $u_{0}(x)$ and $u^{1}(t)$ satisfy the conditions of concordance at the points $x=0, x=1, x=\ell$ :

$$
\left\{\begin{array}{l}
u_{0}(0)=1, \quad u_{0}^{\prime \prime}(0)=0 ; \quad u_{0}(1-0)=u_{0}(1+0), \\
\beta\left[u_{0}^{\prime}\right]_{1+0}^{1-0}+\lambda u_{0}(1)=0 ; \quad \lambda\left[u_{0}^{\prime \prime}\right]_{1-0}^{1+0}=\left(\left[u_{0}^{\prime}\right]_{1+0}^{1-0}\right)^{2}, \\
\lambda u_{0}^{\prime \prime}(1-0)+u_{0}^{\prime}(1-0) \cdot\left[u_{0}^{\prime}\right]_{1+0}^{1-0}=0 ; u_{0}(\ell)=u^{1}(0), u_{0}^{\prime \prime}(\ell)=\dot{u}^{1}(0) .
\end{array}\right.
$$

Lemma 1. Let $u_{0} \in H^{2+\alpha}\left(\overline{\Omega^{ \pm}(0)}\right), u^{1}, \bar{S} \in H^{\frac{2+\alpha}{2}}[0, \infty), 0<\alpha<1, \bar{S}(0)=1$, $\beta \dot{\bar{S}}(0)=-u_{0}(1)$ and $\bar{S}, u_{0}, u^{1}$ satisfy conditions (1.6), (1.7).

Then there exists a unique solution

$$
w \in H^{2+\alpha, \frac{2+\alpha}{2}}\left(\overline{\Omega_{T}^{ \pm}}\right)
$$

of the problem (1.1)-(1.4) with given boundary $\bar{S}(t)$ for all $T>0$.

The proof of this lemma is based upon the methods in [6] and the solvability of the problem

$$
\left\{\begin{array}{l}
\frac{\partial v}{\partial t}=\frac{\partial^{2} v}{\partial x^{2}}, \quad x \neq 0, t>0 ;\left.v\right|_{t=0}=0 \\
{[v]=0, \quad\left[\frac{\partial v}{\partial x}\right]_{+0}^{-0}+a(t) v=b(t), x=0, t>0}
\end{array}\right.
$$

in $R_{T}=\{(x, t)|\cdot| x \mid \leq \infty, 0<t<T\}$ with $a, b, \in H^{\frac{1+\alpha}{2}}[0, T], a(t) \geq 0$.

The problem (1.8) is solved with the help of the problem

$$
\left\{\begin{array}{l}
\frac{\partial v^{+}}{\partial t}=\frac{\partial^{2} v^{+}}{\partial x^{2}}, \quad(x, t) \in R_{T}^{+}=\{x>0,0<t<T\}, \\
\left.v\right|_{t=0}=0,-\frac{\partial v}{\partial x}(0, t)+\frac{a(t)}{2} v(0, t)=\frac{b(t)}{2}, \quad t>0
\end{array}\right.
$$

if we suppose

$$
v(x, t)=\left\{\begin{array}{l}
v^{+}(x, t), \quad x>0, t>0 \\
v^{+}(-x, t), \quad x<0, t>0 .
\end{array}\right.
$$

And finally, the problem (1.9) has a unique solution $v \in H^{2+\alpha, \frac{2+\alpha}{2}}\left(\bar{R}_{T}^{+}\right)$, if $b(0)=0$. This follows from [6].

Theorem 1. Let us assume, that

$$
u_{0} \in H^{2+\alpha}\left(\overline{\Omega^{ \pm}(0)}\right), \quad u^{1} \in H^{\frac{2+\alpha}{2}}[0, \infty)
$$


and $u_{0}, u^{1}$ satisfy conditions (1.7).

Then there exists a unique solution

$$
u \in H^{2+\alpha, \frac{2+\alpha}{2}}\left(\overline{\Omega_{T}^{ \pm}}\right), \quad S \in H^{2+\alpha / 2}[0, T]
$$

of the problem (1.1)-(1.5) with sufficiently small $T>0$.

Proof. Let

$$
\max \left\{\left|u_{0}\right|_{\Omega+(0)}^{(2+\alpha)},\left|u_{0}\right|_{\Omega^{-}(0)}^{(2+\alpha)}, \quad\left|u^{1}\right|_{[0, \infty)}^{\left(\frac{2+\alpha}{2}\right)}\right\} \leq C_{0} .
$$

One can use a fixed point theorem to prove this result. Consider the set

$$
\mathcal{M}=\left\{\bar{u} \in H^{\frac{1+\alpha}{2}}[0, T]|\bar{u}|_{[0, T]}^{\left(\frac{1+\alpha}{2}\right)} \quad \leq 2 C_{0}\right\}
$$

and let

$$
\bar{S}^{2}(t)=1-\frac{2}{\beta} \int_{0}^{t} \bar{u}(\tau) d \tau
$$

with $\bar{u} \in \mathcal{M}$. Choose time $T>0$ such that $\bar{S}(t)$ satisfies condition (1.6) on the interval $[0, T]$.

One can easily show that

$$
T \leq T_{1}=\frac{\beta}{2 C_{0}} \max \{1-\delta, \ell-1-\delta\}
$$

In accordance with lemma 1 the problem (1-1)-(1.4) with $\bar{S}(t)$ given by the formula (1.10) has a unique solution $w(x, t)$ satisfying

$$
|w|_{\Omega_{T}^{+}}^{(2+\alpha)} \leq C_{1}\left(C_{0}, \delta\right) .
$$

The function

$$
\bar{w}(t)=w(\bar{S}(t), t)=F(\bar{u})
$$

defines a compact operator $F$ mapping the set $\mathcal{M}$ into the space $H^{\frac{2+\alpha}{2}}[0, T]$. Hence, if one can choose a time $T$ such that $F$ transform $\mathcal{M}$ into $\mathcal{M}$ then, in accordance with Shauder's fixed point theorem one has proven the existence of at least one classical solution of the problem (1.1) - (1.5).

Since

$$
\bar{w}(t)=\bar{w}(0)+\int_{0}^{t} \bar{w}^{\prime}(\tau) d \tau
$$


and

$$
\frac{|\bar{w}(t+h)-\bar{w}(t)|}{|h|} \leq\left|\bar{w}^{\prime}\left(t_{1}\right)\right|, \quad t_{1} \in[t, t+h]
$$

then

$$
\begin{aligned}
|w|_{[0, T]}^{\frac{(1+\alpha)}{2}} & \leq|\bar{w}(0)|+T|\bar{w}|_{[0, T]}^{(1)}+2 T^{\frac{1-\alpha}{2}}|\bar{w}|_{[0, T]}^{(1)} \leq \\
& \leq C_{0}+C_{1}\left(T+2 T^{\frac{1-\alpha}{2}}\right) \leq 2 C_{0}
\end{aligned}
$$

if

$$
T \leq T_{2}=\left(\frac{C_{0}}{C_{1}\left(T^{\frac{1+\alpha}{2}}+2\right)}\right)^{\frac{2}{1-\alpha}}
$$

Choosing

$$
T \leq \min \left\{T_{1}, T_{2}\right\}
$$

we see that $F$ transforms $\mathcal{M}$ into $\mathcal{M}$.

Before proving the uniqueness of the solution of (1.1)-(1.5) let us transform this problem to a problem on a fixed domain.

The transformations

$$
y=\frac{x}{S(t)}, \quad \tau=t \text { in } \Omega_{T}^{-}
$$

map the domain $\Omega_{T}^{-}$into the domain

$$
\Pi_{T}^{-}=\{(y, \tau) \mid 0<y<1, \quad 0<\tau<T\}
$$

and

$$
y=1+(\ell-1) \frac{x-S(t)}{\ell-S(t)}, \quad \tau=t \text { in } \Omega^{+}(t)
$$

map the domain $\Omega_{T}^{+}$into the domain

$$
\Pi_{T}^{+}=\{(y, \tau) \mid 1<y<\ell, \quad 0<\tau<T\}
$$

The new unknown functions

$$
v(y, \tau) \equiv u(x, t) \text { and } S(\tau)
$$

satisfy the problem similar to $(1.1)-(1.5)$ but in the known domains $\Pi_{T}^{ \pm}$.

If there exist two different solutions of the problem (1.1) - (1.5) then their difference

$$
\bar{v}=v^{1}-v^{2}, \quad \bar{S}=S^{1}-S^{2}
$$


satisfies the following problem

$$
\begin{gathered}
\frac{\partial \bar{v}}{\partial \tau}-\bar{a}^{2} \frac{\partial^{2} \bar{v}}{\partial y^{2}}+\bar{b} \frac{\partial \bar{v}}{\partial y}=A \cdot \bar{S}+B \cdot \frac{d \bar{S}}{d \tau}, \quad(y, \tau) \in \Pi_{T}^{ \pm}, \\
{[v]=0,\left[\bar{a} \frac{\partial \bar{v}}{\partial y}\right]+\frac{\lambda}{\beta} \bar{v}=0, \quad y=1, \tau>0,} \\
\bar{v}=C \cdot \bar{S}+D \cdot \frac{d \bar{S}}{d \tau}, \quad y=1, \tau>0, \\
\bar{v}(0, \tau)=\bar{v}(\ell, \tau)=0, \tau>0, \quad \bar{v}(y, 0)=0, y \in \Omega^{ \pm}(0),
\end{gathered}
$$

with smooth functions $\bar{a}, \bar{b}, A, B, C, D, \bar{a} \geq a_{0}=$ const $>0$.

Applying results similar to those of lemma 1 , one obtains from (1.15), (1.16), (1.18) the following inequality

$$
|\bar{v}|_{\Pi_{T}^{ \pm}}^{(2+\alpha)} \leq C_{2}\left(C_{0}\right)|\bar{S}|_{[0, T]}^{\left(\frac{2+\alpha}{2}\right)}
$$

On the other hand, considering (1.17) as an ordinary differential equation with respect to $\bar{S}$, one can conclude that

$$
|\bar{S}|^{\left(\frac{2+\alpha}{2}\right)}[0, T] \leq C_{3}\left(C_{0}\right)|\bar{v}|_{\prod_{T}}^{(\alpha)}
$$

Applying the simple inequalities

$$
\begin{aligned}
|\bar{v}|_{\Pi_{T}^{+}}^{(\alpha)}= & \left(|\bar{v}|_{\Pi_{T}^{+}}^{(0)}+\left\langle\left.\bar{v}\right|_{\Pi_{T}^{+}} ^{(\alpha)}\right) \leq\right. \\
& T \cdot|\bar{v}|_{\Gamma_{T}^{+}}^{(2+\alpha)}+T^{\frac{2-\alpha}{2}}|\bar{v}|_{\Gamma_{T}^{+}}^{(2+\alpha)}
\end{aligned}
$$

to (1.20) and (1.19) one obtains that

$$
|\bar{S}|_{[0, T]}^{\left(\frac{2+\alpha}{2}\right)} \leq C_{2} C_{3}\left(T+T^{\frac{2-\alpha}{2}}\right)|\bar{S}|_{[0, T]}^{\left(\frac{2+\alpha}{2}\right)}
$$

If

$$
c_{2} c_{3}\left(T+T^{\frac{2-\alpha}{2}}\right)<1
$$

then there only exists the one possibility $\bar{S}(t) \equiv 0$.

Step by step we prove the existence and the uniqueness of the solution (1.1)-(1.5) up to time $T_{*}$ where

$$
\lim _{T \rightarrow T_{*}}|U|_{Q_{T}^{ \pm}}^{(2+\alpha)}=\infty, \text { or } \lim _{t \rightarrow T_{*}} S(t)=0, \ell
$$

One can exclude the case $S\left(T_{*}-0\right)=0, \ell$. Because in this situation after time $T_{*}$ solution of the problem (1.1) - (1.5) is the solution of the heat equation with the boundary condition (1.3) and with the initial data $u\left(x, T_{*}-0\right)$. Hence,

$$
\lim _{T \rightarrow T_{*}}|u|_{\Omega_{T}^{ \pm}}^{(2+\alpha}=\infty, \quad \delta<S(t)<\ell-\delta, t \in\left[0, T_{*}\right] .
$$


Theorem 2. Under assumption theorem $1 T_{*}=\infty$.

Proof. First of all we prove the maximum principle:

$$
\min \left\{\min u^{1}, \min u_{0}\right\} \leq u(x, t) \leq \max \left\{\max u^{1}, \max u_{0}\right\} .
$$

If we suppose that $u(x, t)$ has its absolute positive maximum at the point $\left(S\left(t_{0}\right), t_{0}\right)$ on the free boundary, then

$$
\begin{gathered}
u\left(S\left(t_{0}\right), t_{0}\right)>0 \\
\frac{\partial u}{\partial x}\left(S\left(t_{0}\right)-0, t_{0}\right)>0, \quad \frac{\partial u}{\partial x}\left(S\left(t_{0}\right)+0, t_{0}\right)<0 .
\end{gathered}
$$

But these inequalities contradict the condition (1.2). The same applied to the case of the absolute negative minimum.

The main estimate results from multiplying equation (1.1) by $u_{t}$ and integrating by parts in each domain $\Omega^{ \pm}(t)$ with the help of conditions (1.2) and (1.5):

$$
\begin{gathered}
\int_{\Omega} u_{t}^{2}(x, t) d x+\frac{1}{2} \frac{d}{d t} \int_{\Omega} u_{x}^{2}(x, t) d x+\frac{1}{4} \beta \lambda \frac{d}{d t}\left[\frac{d S^{2}}{d t}\right]^{2}= \\
\frac{1}{2} \frac{d S}{d t} \cdot\left[u_{x}^{2}(S+0, t)-u_{x}^{2}(S-0, t)\right] .
\end{gathered}
$$

Applying the heat equation and the condition (1.2) one has

$$
\begin{aligned}
& \int_{\Omega}\left(u_{t}^{2}+u_{x x}^{2}\right) d x+\frac{d}{d t} \int_{\Omega} u_{x}^{2} d x+\frac{1}{2} \beta \lambda \frac{d}{d t}\left[\frac{d S^{2}}{d t}\right]= \\
& =-\frac{\lambda}{\beta^{2} S(t)} \cdot\left[u_{x}(S+0, t)+u_{x}(S-0, t)\right] \cdot u^{2}(S, t) .
\end{aligned}
$$

This identity, together with the inequality

$$
u_{x}^{2}(S \pm 0, t) \leq C_{4}\left(C_{0}, \delta\right)+\varepsilon \int_{\Omega^{ \pm}(t)} u_{x x}^{2} d x+\frac{C_{5}}{\varepsilon} \int_{\Omega} u_{x}^{2} d x
$$

gives us our main estimate

$$
\iint_{\Omega_{T}^{ \pm}}\left(u_{t}^{2}+u_{x x}^{2}\right) d x d t+\max _{t \in(0, T)} \int_{\Omega} u_{x}^{2} d x \leq C_{6}\left(C_{0}, \delta\right) .
$$

The Sobolev space $W_{2}^{2,1}\left(\Omega_{T}^{ \pm}\right)$is contained in the space $H^{\alpha_{0}, \frac{\alpha_{0}}{2}}\left(\overline{\Omega_{T}^{ \pm}}\right)$with some $\alpha_{0}>0$. Hence, comparing this with condition (1.5) one has that

$$
S \in H^{\frac{2+\alpha_{0}}{2}}[0, T] \text {. }
$$

From lemma 1 it follows that

$$
u \in H^{2+\alpha_{0}, \frac{2+\alpha_{0}}{2}}\left(\bar{\Omega}_{T}^{ \pm}\right)
$$

and reapplying all this once more if $\alpha_{0}<\alpha$, we obtain the final result. 
§. The exact problem (0.16)-(0.19) $(\beta=0)$. The classical formulation of the problem (0.16)-(0.19) has the form:

$$
\begin{gathered}
\frac{\partial u}{\partial t}=\frac{\partial^{2} u}{\partial x^{2}}, \quad x \in \Omega^{ \pm}(t), t>0, \\
{[u]=0, \quad u=0 \quad x=S(t), t>0,} \\
u(0, t)=1, \quad u(\ell, t)=u^{1}(t), t>0 \\
u(x, 0)=u_{0}(x), \quad x \in \Omega, \quad S(0)=1, \\
{\left[u_{x}\right]_{S+0}^{S-0}=\lambda S \cdot \frac{d S}{d t}, \quad x=S(t), t>0 .}
\end{gathered}
$$

In a very similar manner to theorem 1 , one can prove the small-time classical solvability of the problem (2.1)-(2.5).

Theorem 3. Let $u_{0} \in H^{2+\alpha}\left(\overline{\Omega^{ \pm}(0)}\right), u^{1} \in H^{\frac{2+\alpha}{2}}[0, \infty)$ and $u_{0}, u^{1}$ satisfy conditions (1.7) with $\beta=0$.

Then there exists a unique solution

$$
u \in H^{2+\alpha, \frac{2+\alpha}{2}}\left(\overline{\Omega_{T}^{ \pm}}\right), \quad S \in H^{\frac{2+\alpha}{2}}[0, T]
$$

of the problem (2.1) -(2.5), where $T \leq T_{3}$ and $T_{3}$ sufficiently small.

The proof of this theorem is also based upon the fixed point theorem for the set

$$
\mathcal{M}=\left\{\left.\bar{S} \in H^{\frac{2+\alpha}{2}}[0, T]|| \bar{S}\right|_{[0, T]} ^{\left(\frac{2+\alpha}{2}\right)} \leq 2 C_{0}\right\}
$$

where $\bar{S}(t)$ satisfies the condition (1.6). Let $\bar{S}(t)$ be the boundary between the domains $\Omega^{-}(t)$ and $\Omega^{+}(t)$ and $\bar{u}(x, t)$ be the solution of the linear problem (2.1)-(2.4). Then the operator

$$
F(\bar{S}) \equiv\left(1+\frac{2}{\lambda} \int_{0}^{t}\left[\frac{\partial \bar{u}}{\partial x}(\bar{S}(\tau)-0, \tau)-\frac{\partial \bar{u}}{\partial x}(\bar{S}(\tau)+0, \tau)\right] d \tau\right)^{\frac{1}{2}}
$$

maps the set $\mathcal{M}$ into the space $\left.H^{\frac{3+\alpha}{2}}[0, \tau)\right]$, and hence is a compact operator from $\mathcal{M}$ into $H^{\frac{2+\alpha}{2}}[0, T]$. Choosing further the time $T$ sufficiently small, one can show that $F$ transforms $\mathcal{M}$ into $\mathcal{M}$ and, hence, has at least one fixed point.

Uniqueness is proved very similarly to theorem 1 .

The time of existence of classical solution can be controlled with the help fo the distance between the free boundary and the boundary $\{x=0\}$.

Let us explain this by considering the one-phase problem 
Lemma 2. Let $u_{0}$ be the same as in theorem $3, u^{1} \equiv 0, u_{0}(x) \equiv 0$ where $1<x<\ell$ and

$$
\lambda>\frac{9}{4} \int_{0}^{1}\left|u_{0}^{\prime}(x)\right| d x
$$

Then the classical solution of the problem (2.1) - (2.5) exists up to the time $T_{0}$, where

$$
S(t) \geq \frac{9}{4 \lambda} \int_{0}^{1}\left|u_{0}^{\prime}(x)\right| d x=\delta_{0}, \quad 0<t<T_{0}
$$

Proof. One can easily show that

$$
\int_{0}^{S(t)}\left|u_{x}(x, t)\right| d x \leq \int_{0}^{1}\left|u_{0}^{\prime}(x)\right| d x
$$

This estimate is obtained by multiplying equation (2.1) for $q=u_{x}$ by the function

$$
\psi_{\varepsilon}(q)=q\left(q^{2}+\varepsilon^{2}\right)^{-1 / 2},
$$

and integrating by parts and passing to the limit when $\varepsilon \rightarrow 0$.

For the next step, we consider the indentity (1.23) with $\beta=0$ and substitute the derivative $u_{x}(S-0, t)$ from Stefan condition (2.5) instead of $\frac{d S}{d t}$ and the derivative $u_{x x}$ from heat equation instead of the derivative $u_{t}$ :

$$
\int_{0}^{S(t)} u_{x x}^{2} d x+\frac{1}{2} \frac{d}{d t} \int_{0}^{S(t)} u_{x}^{2} d x=\frac{1}{2 \lambda S(t)} \cdot\left|u_{x}^{3}(S-0, t)\right|
$$

Since

$$
\begin{gathered}
\left|u_{x}(S-0, t)\right|^{3 / 2}=\left|u_{x}(0, t)\right|^{3 / 2}+\frac{3}{2} \int_{0}^{S(t)} u_{x x}\left|u_{x}\right|^{1 / 2} d x \leq \\
\quad \leq\left|u_{x}(0, t)\right|^{3 / 2}+\frac{3}{2}\left(\int_{0}^{S} u_{x x}^{2} d x\right)^{1 / 2}\left(\int_{0}^{S}\left|u_{x}\right| d x\right)^{1 / 2} \leq \\
\quad \leq\left|u_{x}(0, t)\right|^{3 / 2}+\frac{3}{2}\left(\int_{0}^{1}\left|u_{0}^{\prime}(x)\right| d x\right)^{\frac{1}{2}} \cdot\left(\int_{0}^{S}\left|u_{x x}^{2}\right| d x\right)^{\frac{1}{2}},
\end{gathered}
$$

then

$$
\begin{gathered}
\int_{0}^{S(t)} u_{x x}^{2} d x+\frac{1}{2} \frac{d}{d t} \int_{0}^{S(t)} u_{x}^{2} d x \leq \frac{1}{\lambda S(t)}\left|u_{x}(0, t)\right|^{3}+ \\
+\frac{9}{4 \lambda S(t)} \cdot \int_{0}^{1}\left|u_{0}^{\prime}(x)\right| d x \cdot \int_{0}^{S(t)} u_{x x}^{2} d x
\end{gathered}
$$


Hence

$$
\iint_{\Omega_{t}^{-}}\left(u_{x x}^{2}+u_{t}^{2}\right) d x d t+\max _{t \in(0, T)} \int_{\Omega^{-}(t)} u_{x}^{2} d x \leq C_{7}\left(C_{0}, \delta_{0}\right)
$$

Using the inequality

$$
\left|u_{x}(S-0, t)\right|^{4} \leq 2\left|u_{x}(0, t)\right|^{4}+\int_{0}^{S(t)} u_{x x}^{2} d x+C_{s}\left(\int_{0}^{S(t)} u_{x}^{2} d x\right)^{3}
$$

and the Stefan condition (2.5) one can obtain that

$$
\int_{0}^{T}\left|\frac{d S}{d t}(t)\right|^{4} d t \leq C_{9}\left(C_{0}, S_{0}\right)
$$

It is known from [6], that the linear problem (2.1)-(2.4) with known boundary $S \in W_{4}^{1}[0, T]$ has a unique solution

$$
u \in W_{4}^{2,1}\left(\Omega_{T}^{-}\right)
$$

The Sobolev space $W_{4}^{2,1}\left(\Omega_{T}^{-}\right)$is contained in the space $H^{1+\alpha_{1}, \frac{1+\alpha_{1}}{2}}\left(\overline{\Omega_{T}^{-}}\right)$. Hence from the Stefan condition one has

$$
S \in H^{\frac{2+\alpha_{1}}{2}}[0, T], \quad 0<\alpha_{1}<\frac{1}{4}
$$

Returning to the linear problem (2.1)-(2.4) with known boundary $x=S(t)$ in the space $H^{\frac{2+\alpha_{1}}{2}}[0, T]$ one can see as in [6] that

$$
u \in H^{2+\alpha_{2}, \frac{2+\alpha_{2}}{2}}\left(\overline{\Omega_{T}^{-}}\right), \quad \alpha_{2}=\min \left(\alpha, \alpha_{1}\right)
$$

If $\alpha_{1}<\alpha$ then reapplying the above, one obtains the final result.

LEMma 3. Let $u^{1} \in H^{\frac{1+\alpha}{2}}[0, \infty), u_{0}, u^{\beta} \in H^{2+\alpha}\left(\overline{\Omega^{ \pm}(0)}\right), u^{1}, u_{0}^{\beta}$ satisfy the conditions (1.7) with $\beta>0, u^{1}, u_{0}$ satisfy the conditions (1.7) with $\beta=0$ and

$$
\lim _{\beta \rightarrow 0}\left|u_{0}^{\beta}-u_{0}\right|_{\Omega^{ \pm}(0)}^{(2+\alpha)}=0
$$

Then the solution $\left\{u^{\beta}(x, t), S^{\beta}(t)\right\}$ of the problem (1.1) - (1.5) with the data $\left\{u^{1}, u_{0}^{\beta}\right\}$ converges to the problem (2.1) - (2.5) with data $\left\{u^{1}, u_{0}\right\}$ as $\beta \rightarrow 0$ in $H^{2+\alpha, \frac{2+\alpha}{2}}\left(\bar{\Omega}_{T}^{ \pm}\right)$, where $T \leq T_{4}$ and $T_{4}$ is sufficiently small.

Proof. Let us consider the identity (1.23) and substitute the derivatives $u_{x}^{\beta}\left(S^{\beta} \pm 0, t\right)$ from Stefan condition (2.5) instead of $\frac{d S}{d t}$. (note, that $u^{\beta}(x, t)$ satisfies the condition (2.5)). Further estimating the right hand side of this identity with the help of the inequality

$$
\begin{aligned}
& \left.\left|u_{x}^{\beta}(S(t) \pm 0, t)\right|^{3} \leq C_{10}+3 \int_{\Omega^{ \pm}(t)}\left|u_{x x}^{\beta}\right|^{2} d x\right)^{1 / 2} \cdot\left(\int_{\Omega^{ \pm}(t)}\left|u_{x}^{\beta}\right|^{4} d x\right)^{1 / 2} \leq \\
& \leq C_{10}+\varepsilon \int_{\Omega^{ \pm}(t)}\left|u_{x x}^{\beta}\right|^{2} d x+C_{11}\left(C_{0}, \varepsilon\right)\left(\int_{\Omega^{ \pm}(t)}\left|u_{x x}^{\beta}\right|^{2} d x\right)^{3}
\end{aligned}
$$


and using the heat equation one obtains that

$$
\begin{gathered}
\int_{\Omega}\left\{\left|u_{x x}^{\beta}\right|^{2}+\left|u_{t}^{\beta}\right|^{2}\right\} d x+\frac{d}{d t}\left\{\int_{\Omega}\left|u_{x}^{\beta}\right|^{2} d x+\lambda \beta\left|\frac{d\left|S^{\beta}\right|^{2}}{d t}\right|^{2}\right\} \leq \\
\leq C_{12}\left(C_{0}, \delta\right)+C_{13}\left(C_{0}, \delta\right)\left(\int_{\Omega}\left|u_{x}^{\beta}\right|^{2} d x\right)^{3}
\end{gathered}
$$

and

$$
\iint_{\Omega_{T}}\left\{\left|u_{x x}^{\beta}\right|^{2}+\left|u_{t}^{\beta}\right|^{2}\right\} d x d t+\max _{0<t<T} \int_{\Omega}\left|u_{x}^{\beta}(x, t)\right| d x \leq C_{14}\left(C_{0}, \delta\right)
$$

for $T \leq T_{4}\left(C_{0}, \delta\right)$.

Similarly to lemma 2 one can show that

$$
S^{\beta} \in W_{4}^{1}[0, T]
$$

The estimates $(2.12),(2.13)$, allow one to choose subsequence $\left\{u^{\beta k}(x, t), S^{\beta_{k}}(t)\right\}$ which converges weakly in $W_{2}^{2,1}\left(\Omega_{T}^{ \pm}\right) \otimes W_{4}^{1}[0, T]$ to the solution

$$
\bar{u} \in W_{2}^{2,1}\left(\Omega_{T}^{ \pm}\right), \quad \bar{S} \in W_{4}^{1}[0, T]
$$

of the problem $(2.1)-(2.5)$ as $\beta \rightarrow 0$. Almost identically to lemma 2 , one can show that

$$
\bar{u} \in H^{2+\alpha, \frac{2+\alpha}{2}}\left(\overline{\Omega_{T}^{ \pm}}\right), \bar{S} \in H^{\frac{2+\alpha}{2}}[0, T]
$$

and, hence, it coincides with the unique classical solution $\{u(x, t), S(t)\}$ corresponding to the data $\left\{u_{0}, u^{1}\right\}$.

Let us prove now that problem (0.16) - (0.19) has at least one global-time weak solution with monotone free boundary. This corresponds to the case of a melting solid ball.

THEOREM 4. Under the assumptions of theorem 3 let $u_{0}(x) \geq 0, u_{0}(x) \equiv 0$ when $x \in \Omega^{-}(0)$ and $u^{1}(t) \equiv 0$.

Then there exists at least one weak solution $\{u(x, t), \varphi(x, t)\}$ of the problem $(0.16)$ (0.19) such that $\varphi \equiv 0$ in the domain

$$
\Omega_{\infty}^{-}=\{(x, t) \mid \quad 0<x<\ell, \quad 0<t<g(x)\}
$$

and $\varphi \equiv 1$ in the domain

$$
\Omega_{\infty}^{+}=\{(x, t) \mid 0<x<\ell, g(x)<t<\infty\}
$$


where $g(x)$ is a continuous monotone function on the interval $\left(X_{*}, \ell\right), g(x) \equiv 0$ when $x \in(1, \ell)$,

$$
\begin{gathered}
\lim _{x \rightarrow X_{*}+0} g(x)=\infty \text { and } g(x)=\infty \text { when } x \in\left[0, X_{*}\right) \text { if } \\
X_{*}>0(g(x) \text { may be bounded for all } x \in[0, \ell))
\end{gathered}
$$

Proof. Let the $u_{0}^{\beta}(x)$ be nonnegative sufficiently smooth functions satisfying conditions (1.7) with $\beta>0$ and converging to $u_{0}(x)$ in $H^{2+\alpha}\left(\overline{\Omega^{ \pm}(0)}\right)$. In accordance with theorem 2 there exists a unique classical solution $\left\{u^{\beta}(x, t), \bar{S}^{\beta}(t)\right\}$, where $\bar{S}^{\beta}=S^{\beta}(t)$ when $0<$ $S^{\beta}(t)<\ell$ and $\bar{S}^{\beta} \equiv 0, \ell, t>T_{*}^{\beta}$, when $S^{\beta}\left(T_{*}^{\beta}\right)=0, \ell$.

Let $g^{\beta}(x)$ be the inverses of the functions $S^{\beta}(t)$ on the interval $\left(0, T_{*}^{\beta}\right)$ :

$$
x \equiv S^{\beta}\left(g^{\beta}(x)\right) .
$$

If $T_{*}^{\beta}=\infty$, then suppose $g^{\beta}(x)=\infty$ when $x \in\left(0, X_{*}^{\beta}\right), X_{*}^{\beta}=\lim _{t \rightarrow \infty} S^{\beta}(t)$.

Suppose $\varphi^{\beta}(x, t)=0$ in the domain $\Omega_{\beta, \infty}^{-}=\left\{(x, t) \mid 0<x<\ell, 0<t<g^{\beta}(x)\right\}$ and $\varphi^{\beta}(x, t)=1$ in the domain $\Omega_{\beta, \infty}^{+}=\left\{(x, t) \mid 0<x<\ell, g^{\beta}(x)<t<\infty\right\}$. For all $\beta>0$ the functions $u^{\beta}, \varphi^{\beta}$ satisfy the identity

$$
\begin{gathered}
\left.\int_{\Omega}\left(u^{\beta}+\lambda x \varphi^{\beta}\right) \eta\right|_{t=T} d x+\iint_{\Omega_{T}}\left\{-\left(u^{\beta}+\lambda x \varphi^{\beta}\right) \eta_{t}+u_{x}^{\beta} \cdot \eta_{x}\right\} d x d t= \\
\quad=\left.\int_{\Omega}\left(u_{0}^{\beta}+\lambda x \varphi_{0}^{\beta}\right) \eta\right|_{t=0} d x+\int_{0}^{T}\left(\eta u_{x}^{\beta}\right)_{x=\ell} d t
\end{gathered}
$$

where $\eta$ is an arbitrary smooth function such that $\eta(0, t)=0$.

The maximum principle gives us

$$
\begin{gathered}
0 \leq u^{\beta}(x, t) \leq \max \left\{1, \max \left|U_{0}^{\beta}\right|\right\}=A_{0} \\
-\frac{1}{\beta S^{\beta}(t)} \cdot u^{\beta}\left(S^{\beta}(t), t\right)=\frac{d S^{\beta}}{d t}(t) \leq 0, \\
0 \leq S^{\beta}(t) \leq 1 .
\end{gathered}
$$

From (2.17), lemma 3 and local estimates for linear equation from [6] it follows that in the domain $G=\{(x, t) \mid 1<x<\ell\}$

$$
\left|u^{\beta}\right|_{G}^{(2+\alpha)} \leq A_{1}\left(C_{0}\right)
$$

If in (2.14) $\eta=x u^{\beta}(x, t)$ then one can easily obtain that

$$
\iint_{\Omega_{T}} x\left|u_{x}^{\beta}\right|^{2} d x d t \leq A_{2}\left(C_{0}\right) .
$$


The estimates (2.15)-(2.19) allow one to choose subsequences $\left\{u^{\beta_{k}}(x, t)\right\},\left\{g^{\beta_{k}}(x)\right\}$, $\left\{\varphi^{\beta_{k}}(x, t)\right\}$ such that when $\beta \rightarrow 0 g^{\beta_{k}}(x)$ converges to a monotone function $g(x)$ almost everywhere; $\varphi^{\beta_{k}}(x, t)$ converges to a function $\varphi(x, t)$ almost everywhere; and $u^{\beta_{k}}(x, t)$ converges to a function $u(x, t)$ weakly in $\tilde{W}_{2}^{1,0}\left(\Omega_{T}\right)$, where

$$
\tilde{W}_{2}^{1,0}\left(\Omega_{T}\right)=\left\{w \mid \iint_{\Omega_{T}}\left(w^{2}+x w_{x}^{2}\right) d x d t<\infty\right\} .
$$

Moreover $u^{\beta_{k}}(x, t)$ converges to $u(x, t)$ in

$$
H^{2+\alpha, \frac{2+\alpha}{2}}\left(\bar{\Omega}_{T_{4}}^{ \pm}\right) \cap H^{2+\alpha, \frac{2+\alpha}{2}}(\bar{G})
$$

and $S^{\beta_{k}}(t)$ converges to a monotone function $S(t)$ almost everywhere. The functions $u(x, t)$ and $\varphi(x, t)$ satisfy the identity (2.14) and $u(x, t)$ satisfies the condition (2.2) in the following sense

$$
u\left(x_{0}, t\right)=\int_{S(t)}^{x_{0}} u_{x}(x, t) d x
$$

for almost every $t \in\left[0, T_{*}\right], T_{*}=\lim _{\beta_{k} \rightarrow 0} T_{*}^{\beta_{k}}$.

The proof of the equality (2.20) is based upon the identity

$$
\begin{gathered}
\iint_{\Omega_{T}} \eta(t) u_{x}^{\beta} \varphi^{\beta} d x d t=\beta \int_{0}^{T} \eta S^{\beta} \frac{d S^{\beta}}{d t} d t= \\
=-\frac{\beta}{2} \int_{0}^{T}\left(S^{\beta}\right)^{2} \cdot \frac{d \eta}{d t} d t
\end{gathered}
$$

where $\eta(t)$ is an arbitrary smooth function satisfying conditions $\eta(0)=\eta(T)=0$. If one passes to the limit as $\beta \rightarrow 0$ then

$$
\int_{0}^{T} \eta(t)\left\{\int_{S(t)}^{\ell} u_{x}(x, t) d x\right\} d t=0,
$$

and, hence

$$
\int_{S(t)}^{\ell} u_{x}(x, t) d x=0
$$

for almost every $t \in\left[0, T_{*}\right]$. The last equality is equivalent to $(2.20)$ if $x_{0}>S(t)$. The case $x_{0}<S(t)$ is handled similarly.

Now, let us show that $g(x)$ is a continuous function on the interval $\left(X_{*}, 1\right)$.

If $g(x)$ is discontinuous at some point $\bar{x} \in\left(X_{*}, 1\right)$, then there exists a positive constants $h, \bar{t}$ such that the domain $Q^{-}=\{(x, t) \mid \bar{x}-h<x<\bar{x}, \dot{\bar{t}}<t<\bar{t}+h\}$ is contained in $\Omega_{\infty}^{-}$ 
and the domain $Q^{+}=\{(x, t) \mid \bar{x}<x<\bar{x}+h, \bar{t}<t<\bar{t}+h\}$ is contained in $\Omega_{\infty}^{+}$. From (2.14) it follows that

$$
\begin{gathered}
\frac{\partial u}{\partial t}=\frac{\partial^{2} u}{\partial x^{2}} \text { in } \overline{Q^{-}} \cup \overline{Q^{+}} \\
u(\bar{x}-h, t)>0, \quad u(\bar{x}+h, t)>0, \quad t \in[\bar{t}, \bar{t}+h], \\
u(x, \bar{t}) \geq 0, \quad x \in[\bar{x}-h, \bar{x}+h] .
\end{gathered}
$$

and from (2.20) it follows that

$$
u(\bar{x}, t)=0, \quad t \in[\bar{t}, \bar{t}+h] .
$$

The last equality contradicts the maximum principle.

Hence $g(x)$ is a continuous function and the monotone continuous functions $g^{\beta}(x)$ uniformly converge to $g(x)$ on the interval $\left[x_{*}, 1\right]$.

It is known from lemma 3 that the weak solution $\{u, \varphi\}$ is actually a classical solution on the small interval $\left[0, T_{4}\right]$.

Now we prove that this solution cannot be a classical solution for large time.

More precisely we prove that $S(t)$ is discontinuous at some point $t_{*}$.

Let $t_{*}$ be such that $g(x)$ is strictly monotone on the interval $\left(x_{*}, 1\right)$, where $g\left(x_{*}\right)=t_{*}$ (or $S(t)$ is strictly monotone on the interval $\left(0, t_{*}\right)$ ). We prove that $x_{*}>X_{*}$ if $g(x)$ is unbounded and $x_{*}>0$ if $g(x)$ is bounded.

The last statement is equivalent to the discontinuity of the function $S(t)$.

First of all we prove that in the domain $\Omega_{T}^{+}=\{(x, t) \mid S(t)<x<\ell, 0<t<T\}$, $T<t *$ we have $u(x, t) \equiv 0$.

If $\bar{u}=\varphi u$, then $\bar{u} \in \stackrel{\circ}{W}_{2}^{1,0}\left(\Omega_{T}^{+}\right)$and

$$
\left.\int_{S(T)}^{\ell} \bar{u} \eta\right|_{t=T} d x+\iint_{\Omega_{T}^{+}}\left(-\bar{u} \eta_{t}+\bar{u}_{x} \eta_{x}\right) d x d t=0
$$

with $\eta \in \stackrel{\circ}{W}_{2}^{1,1}\left(\bar{\Omega}_{T}^{+}\right)$an arbitrary function.

In the new variables

$$
\tau=t, \quad y=x-S(t)
$$

the functions

$$
v(y, \tau) \equiv u(x, t), \quad \zeta(y, \tau) \equiv \eta(x, t)
$$

satisfy the identity

$$
\begin{aligned}
& \int_{0}^{T}\left(\int_{0}^{\ell-S(\tau)}\left(-v \zeta_{\tau}+v_{y} \zeta_{y}\right) d y\right) d \tau+\left.\int_{0}^{\ell-s(\tau)} v \zeta\right|_{\tau=T} d y= \\
& =-\int_{0}^{T}\left(\int_{0}^{\ell-S(\tau)} v \zeta_{y} d y\right) d S(\tau) .
\end{aligned}
$$


The integral on the right hand side of the inequality (2.23) makes sense since $\dot{S}$ is a measure.

Let

$$
v_{h}=\frac{1}{h} \int_{\tau-h}^{\tau} v(y, t) d t, \quad v_{h}=\frac{1}{h} \int_{\tau}^{\tau+h} v(y, t) d t,
$$

and

$$
\zeta=\left(v_{h}\right)_{h} .
$$

Since

$$
\int_{0}^{T} v \omega_{h} d \tau=\int_{0}^{T} v_{h} \cdot \omega d \tau
$$

then after some transformations (2.23) one obtains that

$$
\begin{aligned}
& \frac{1}{2} \int_{0}^{\ell-S(T)}\left|v_{\bar{h}}(y, T)\right|^{2} d y+\int_{0}^{T}\left(\int_{0}^{\ell-S(\tau)}\left|v_{\bar{h} y}\right|^{2} d y\right) d \tau= \\
& \quad=\left.\int_{0}^{T}\left(v_{y} v_{\bar{h} h}\right)\right|_{y=\ell-S(\tau)} d \tau-\left.\frac{1}{2} \int_{0}^{T}\left(v_{\bar{h}}\right)^{2}\right|_{y=\ell-S(\tau)} d S(\tau) .
\end{aligned}
$$

Passing to the limit as $h \rightarrow 0$ and taking into account that $v(y, \tau)$ sufficiently smooth near $y=\ell-S(\tau)$ and that $v=0$ when $y=\ell-S(\tau)$ one has that

$$
\begin{aligned}
& v(y, \tau) \equiv 0 \text { when } 0<y<\ell-S(\tau), \quad 0<\tau \leq T \leq t_{*} . \\
& \text { Hence } u(x, t) \equiv 0 \text { in } \Omega_{T_{*}}^{+} .
\end{aligned}
$$

If in (2.14) $\eta=x$ then this identity is equivalent to the identity (0.27). Hence the next statement is valid.

ThEOREM 5. Under the assumptions of theorem 4, let $\lambda$ satisfy the condition (0.28). Then the weak solution of the problem (0.16) - (0.19) cannot be a classical solution: there exists a point $\bar{t}_{*}$ such that

$$
S\left(\bar{t}_{*}+0\right)<S\left(\bar{t}_{*}-0\right) .
$$

Now our last result.

Lemma 4. Let $\ell=1, \varphi_{0}=0, u^{1} \equiv 0$. Then the problem $(0.16)-(0.19)$ has at least two different solutions.

Proof. The first solution is the limit of the solutions of the problem $(0.16)-(0.19)$ with $\ell>1, u_{0}(x) \equiv 0, x \in(1, \ell) ; \varphi_{0} \equiv 0, x \in(0,1), \varphi_{0} \equiv 1, x \in(1, \ell)$ as $\ell \rightarrow 1$.

The second solution is the solution $\stackrel{\circ}{u}(x, t)$ of the heat equation in the domain $\Omega_{T}$ with corresponding initial and boundary data and $\stackrel{\circ}{\varphi}(x, t) \equiv 0$ in $\Omega_{T}$, that is the medium is in the solid phase at every point and for all time. 
Remark. About the question of J. Ockendon and S. Howison: let $t_{0}$ be the time of disappearance of the solid ball and the solution of the problem $(0.24)-(0.26)$ is classical for $t<t_{0}$. Then we get from $(0.27)$

$$
t_{0}=\frac{\lambda}{3}-\int_{0}^{1} x \cdot u_{0}(x) d x
$$

I would like to thank J. Ockendon, S. Howison and K. Promislow for fruitful discussions.

\section{REFERENCES}

[1] A. Visintin, Models for supercooling and superheating effects, Research Notes in Mathematics, 120, Pitman Publishing Inc. (1985), pp. 200 - 207.

[2] S. Luckhaus, Solutions for the two-phase Stefan problem with the Gibbs-Thomson Law for the melting temperature, Euro. Jnl. of Applied Mathematics, 1 (1990), pp. 101-111.

[3] X. Chen, F. Reitich, Local existence and uniqueness of solutions of the Stefan problem with surface tension and kinetic undercooling, IMA Preprint Series, 715 (November 1990).

[4] E. RadKevitch, Gibbs-Thomson law and existence of the classical solution of the modified Stefan problem, Soviet Dokl. of Academ. of Science, v. 315 N. 6 (1991).

[5] J. Chadam, S. Howison, P. Ortoleva, Existence and Stability for Spherical Crystal Growing in a Supersaturated Solution, IMA Journal of Appl. Math., 39 (1987), pp. 1-15.

[6] O.A. Ladyzenskaya, V.A. Solonnikov, N.N. Ural'ceva, Linear and Quasilinear Equations of Parabolic Type, Amer. Math. Soc. Transl., American Mathematical Society, Providence, R.I. (1968). 


\section{Recent IMA Preprints \\ Title}

Ian M. Anderson, Niky Kamran and Peter J. Olver, Internal, external and generalized symmetries C. Foias and J.C. Saut, Asymptotic integration of Navier-Stokes equations with potential forces. I

Ling Ma, The convergence of semidiscrete methods for a system of reaction-diffusion equations

Adelina Georgescu, Models of asymptotic approximation

A. Makagon and H. Salehi, On bounded and harmonizable solutions on infinite order arma systems San-Yih Lin and Yan-Shin Chin, An upwind finite-volume scheme with a triangular mesh for conservation laws J.M. Ball, P.J. Holmes, R.D. James, R.L. Pego \& P.J. Swart, On the dynamics of fine structure KangPing Chen and Daniel D. Joseph, Lubrication theory and long waves

J.L. Ericksen, Local bifurcation theory for thermoelastic Bravais lattices

Mario Taboada and Yuncheng You, Some stability results for perturbed semilinear parabolic equations

A.J. Lawrance, Local and deletion influence

Bogdan Vernescu, Convergence results for the homogenization of flow in fractured porous media

Xinfu Chen and Avner Friedman, Mathematical modeling of semiconductor lasers

Yongzhi Xu, Scattering of acoustic wave by obstacle in stratified medium

Songmu Zheng, Global existence for a thermodynamically consistent model of phase field type

Heinrich Freistühler and E. Bruce Pitman, A numerical study of a rotationally degenerate hyperbolic system part I: the Riemann problem

Epifanio G. Virga, New variational problems in the statics of liquid crystals

Yoshikazu Giga and Shun'ichi Goto, Geometric evolution of phase-boundaries

Ling Ma, Large time study of finite element methods for 2D Navier-Stokes equations

Mitchell Luskin and Ling Ma, Analysis of the finite element approximation of microstructure in micromagnetics

M. Chipot, Numerical analysis of oscillations in nonconvex problems

J. Carrillo and M. Chipot, The dam problem with leaky boundary conditions

Eduard Harabetian and Robert Pego, Efficient hybrid shock capturing schemes

B.L.J. Braaksma, Multisummability and Stokes multipliers of linear meromorphic differential equations

Tae Il Jeon and Tze-Chien Sun, A central limit theorem for non-linear vector functionals of vector Gaussian processes

Chris Grant, Solutions to evolution equations with near-equilibrium initial values

Mario Taboada and Yuncheng You, Invariant manifolds for retarded semilinear wave equations

Peter Rejto and Mario Taboada, Unique solvability of nonlinear Volterra equations in weighted spaces

Hi Jun Choe, Holder regularity for the gradient of solutions of certain singular parabolic equations

Jack D. Dockery, Existence of standing pulse solutions for an excitable activator-inhibitory system

Jack D. Dockery and Roger Lui, Existence of travelling wave solutions for a bistable evolutionary ecology model

Giovanni Alberti, Luigi Ambrosio and Giuseppe Buttazzo, Singular perturbation problems with a compact support semilinear term

Emad A. Fatemi, Numerical schemes for constrained minimization problems

Y. Kuang and H.L. Smith, Slowly oscillating periodic solutions of autonomous state-dependent delay equations

Emad A. Fatemi, A new splitting method for scaler conservation laws with stiff source terms

Hi Jun Choe, A regularity theory for a more general class of quasilinear parabolic partial differential equations and variational inequalities

Haitao Fan, A vanishing viscosity approach on the dynamics of phase transitions in Van Der Waals fluids

T.A. Osborn and F.H. Molzahn, The Wigner-Weyl transform on tori and connected graph propagator representations

Avner Friedman and Bei Hu, A free boundary problem arising in superconductor modeling

Avner Friedman and Wenxiong Liu, An augmented drift-diffusion model in semiconductor device

Avner Friedman and Miguel A. Herrero, Extinction and positivity for a system of semilinear parabolic variational inequalities

David Dobson and Avner Friedman, The time-harmonic Maxwell equations in a doubly periodic structure

Hi Jun Choe, Interior behaviour of minimizers for certain functionals with nonstandard growth

Vincenzo M. Tortorelli and Epifanio G. Virga, Axis-symmetric boundary-value problems for nematic liquid crystals with variable degree of orientation

Nikan B. Firoozye and Robert V. Kohn, Geometric parameters and the relaxation of multiwell energies

Haitao Fan and Marshall Slemrod, The Riemann problem for systems of conservation laws of mixed type

Joseph D. Fehribach, Analysis and application of a continuation method for a self-similar coupled Stefan system

C. Foias, M.S. Jolly, I.G. Kevrekidis and E.S. Titi, Dissipativity of numerical schemes

D.D. Joseph, T.Y.J. Liao and J.-C. Saut, Kelvin-Helmholtz mechanism for side branching in the displacement of light with heavy fluid under gravity 
Chris Grant, Solutions to evolution equations with near-equilibrium initial values

B. Cockburn, F. Coquel, Ph. LeFloch and C.W. Shu, Convergence of finite volume methods

N.G. Lloyd and J.M. Pearson, Computing centre conditions for certain cubic systems

João Palhoto Matos, Young measures and the absence of fine microstructures in the $\alpha-\beta$ quartz phase transition

L.A. Peletier \& W.C. Troy, Self-similar solutions for infiltration of dopant into semiconductors

H. Scott Dumas and James A. Ellison, Nekhoroshev's theorem, ergodicity, and the motion of energetic charged particles in crystals

Stathis Filippas and Robert V. Kohn, Refined asymptotics for the blowup of $u_{t}-\Delta u=u^{p}$.

Patricia Bauman, Nicholas C. Owen and Daniel Phillips, Maximum principles and a priori estimates for an incompressible material in nonlinear elasticity

Patricia Bauman, Nicholas C. Owen and Daniel Phillips, Maximal smoothness of solutions to certain Euler-Lagrange equations from nonlinear elasticity

Jack Carr and Robert Pego, Self-similarity in a coarsening model in one dimension

J.M. Greenberg, The shock generation problem for a discrete gas with short range repulsive forces

George R. Sell and Mario Taboada, Local dissipativity and attractors for the Kuramoto-Sivashinsky equation in thin $2 \mathrm{D}$ domains

T. Subba Rao, Analysis of nonlinear time series (and chaos) by bispectral methods

Nicholas Baumann, Daniel D. Joseph, Paul Mohr and Yuriko Renardy, Vortex rings of one fluid in another free fall

Oscar Bruno, Avner Friedman and Fernando Reitich, Asymptotic behavior for a coalescence problem

Johannes C.C. Nitsche, Periodic surfaces which are extremal for energy functionals containing curvature functions

F. Abergel and J.L. Bona, A mathematical theory for viscous, free-surface flows over a perturbed plane Gunduz Caginalp and Xinfu Chen, Phase field equations in the singular limit of sharp interface problems Robert P. Gilbert and Yongzhi Xu, An inverse problem for harmonic acoustics in stratified oceans Roger Fosdick and Eric Volkmann, Normality and convexity of the yield surface in nonlinear plasticity H.S. Brown, I.G. Kevrekidis and M.S. Jolly, A minimal model for spatio-temporal patterns in thin film flow Chao-Nien Chen, On the uniqueness of solutions of some second order differential equations

Xinfu Chen and Avner Friedman, The thermistor problem for conductivity which vanishes at large temperature Xinfu Chen and Avner Friedman, The thermistor problem with one-zero conductivity

E.G. Kalnins and W. Miller, Jr., Separation of variables for the Dirac equation in Kerr Newman space time E. Knobloch, M.R.E. Proctor and N.O. Weiss, Finite-dimensional description of doubly diffusive convection V.V. Pukhnachov, Mathematical model of natural convection under low gravity

M.C. Knaap, Existence and non-existence for quasi-linear elliptic equations with the p-laplacian involving critical Sobolev exponents

Stathis Filippas and Wenxiong Liu, On the blowup of multidimensional semilinear heat equations

A.M. Meirmanov, The Stefan problem with surface tension in the three dimensional case with spherical symmetry: non-existence of the classical solution

Bo Guan and Joel Spruck, Interior gradient estimates for solutions of prescribed curvature equations of of parabolic type

Hi Jun Choe, Regularity for solutions of nonlinear variational inequalities with gradient constraints

Peter Shi and Yongzhi Xu, Quasistatic linear thermoelasticity on the unit disk

Satyanad Kichenassamy and Peter J. Olver, Existence and non-existence of solitary wave solutions to higher order model evolution equations

Dening Li, Regularity of solutions for a two-phase degenerate Stefan Problem

Marek Fila, Bernhard Kawohl and Howard A. Levine, Quenching for quasilinear equations

Yoshikazu Giga, Shun'ichi Goto and Hitoshi Ishii, Global existence of weak solutions for interface equations coupled with diffusion equations

Mark J. Friedman and Eusebius J. Doedel, Computational methods for global analysis of homoclinic and hetero clinic orbits: a case study

Mark J. Friedman, Numerical analysis and accurate computation of heteroclinic orbits in the case of center manifolds

809 Peter W. Bates and Songmu Zheng, Inertial manifolds and inertial sets for the phase-field equations

810 J. López Gómez, V. Márquez and N. Wolanski, Global behavior of positive solutions to a semilinear equation with a nonlinear flux condition

811 Xinfu Chen and Fahuai Yi, Regularity of the free boundary of a continuous casting problem

812 Eden, A., Foias, C., Nicolaenko, B. and Temam, R., Inertial sets for dissipative evolution equations Part I: Construction and applications

813 Jose-Francisco Rodrigues and Boris Zaltzman, On classical solutions of the two-phase steady-state Stefan problem in strips

814 Viorel Barbu and Srdjan Stojanovic, Controlling the free boundary of elliptic variational inequalities on a variable domain 